\title{
Bloody Discharge
}

National Cancer Institute

\section{Source}

National Cancer Institute. Bloody Discharge. NCI Thesaurus. Code C121557.

The leaking of serosanguinous fluids from an orifice or wound. 